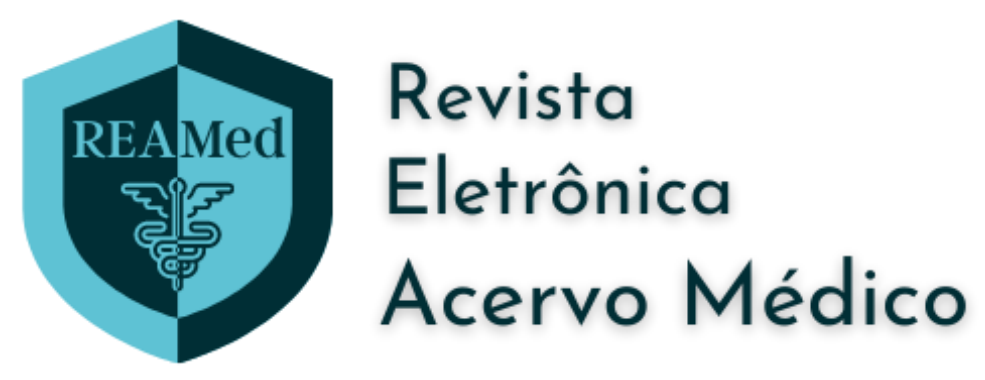

\title{
Abordagem geral da asma: uma revisão narrativa
}

\author{
General approach to asthma: a narrative review
}

Abordaje general del asma: una revisión narrativa

Amanda Santos Rodrigues ${ }^{1}$, Lâmia André Sobrinho², Bianca Danielle Ferreira ${ }^{2}$, Sarah Mendonça Mota $^{2}$, Isabela Coelho Cardoso ${ }^{2}$, Marcela Romano Rahal ${ }^{2}$, Bianca Ridolfi Melchiori ${ }^{3}$, Ana Laura de Lima Rossi ${ }^{3}$, Lais Siqueira Moreira ${ }^{4}$, Felipe Kazuyoshi Miura ${ }^{3}$.

\section{RESUMO}

Objetivo: Relatar de forma atualizada uma abordagem geral da asma. Revisão bibliográfica: A asma pode ser definida como uma condição causada por broncoconstrições episódicas e reversíveis, secundária a inflamação da via aérea, que pode ocorrer por vários fatores, como infecções, alérgenos ambientais e irritantes. É uma condição imunomediada, multifatorial que se apresenta com uma clínica bastante variada e complexa. A inflamação alérgica é comandada pelos linfócitos T auxiliares CD4 + (Th2), os quais secretam interleucina (IL)-4, IL-5 e IL-13. O diagnóstico é feito na presença de fatores de risco para a doença, sinais e sintomas respiratórios típicos de limitação aguda e reversível do fluxo de ar documentada por espirometria. Os medicamentos usados no tratamento do paciente asmático são divididos em dois grupos: os de alívio e os controladores. Como representantes dos fármacos de alívio têm os B2 agonistas de curta duração, anticolinérgicos e corticosteroides sistêmicos, enquanto que os controladores são, principalmente, os B2 agonistas de longa duração e os glicocorticoides inalatórios. Considerações finais: A asma é uma condição que repercute na vida diária do paciente. Sua fisiopatologia está relacionada à produção de citocinas inflamatórias que desencadeiam a crise asmática, que é classificada e tratada quando analisa-se quadro clínico e espirometria.

Palavras-chave: Asma, Obstrução, Exacerbação.

\footnotetext{
ABSTRACT

Objective: Report an updated general approach to asthma. Bibliographic review: Asthma can be defined as a condition caused by episodic and reversible bronchoconstriction, secondary to airway inflammation, which can occur due to several factors, such as infections, environmental allergens and irritants. It is an immunemediated, multifactorial condition that presents with a very varied and complex clinic. Allergic inflammation is commanded by CD4 + helper T lymphocytes (Th2), which secretes interleukin (IL)-4, IL-5 and IL-13. Diagnosis

${ }^{1}$ Centro universitário Christus (UNICHRISTUS), Fortaleza - CE. *E-mail: amanda.taby@gmail.com

${ }^{2}$ Centro Universitário Municipal de Franca (Uni-FACEF), Franca - SP.

${ }^{3}$ Universidade Municipal de São Caetano do Sul (USCS), São Caetano do Sul - SP.

${ }^{4}$ Universidade Anhembi Morumbi (UAM), Morumbi - SP.
} 
is made in the presence of risk factors for the disease, respiratory signs and symptoms typical of acute and reversible airflow limitation documented by spirometry. Medications used to treat asthmatic patients are divided into two groups: relief and controllers. As representatives of relief drugs, we have short-acting B2 agonists, anticholinergics and systemic corticosteroids, while the controllers are mainly long-acting B2 agonists and inhaled glucocorticoids. Final considerations: Asthma is a condition that affects the patient's daily life. Its pathophysiology is related to the production of inflammatory cytokines that trigger an asthma attack, which is classified and treated when analyzing the clinical picture and spirometry.

Key words: Asthma, Obstruction, Exacerbation.

\section{RESUMEN}

Objetivo: Informar un abordaje general actualizado del asma. Revisión bibliográfica: El asma está definido como una condición causada por broncoconstricciones episódicas y reversibles, secundarias a la inflamación de vías respiratorias, que puede ocurrir debido a varios factores, como infecciones, alérgenos ambientales e irritantes. Es una enfermedad multifactorial inmunomediada que se presenta con una clínica muy variada y compleja. La inflamación alérgica está comandada por linfocitos T auxiliares CD4 + (Th2), que secretan interleucina (IL) -4, IL-5 e IL-13. El diagnóstico se basa en los factores de riesgo, signos y síntomas respiratorios propios de la limitación aguda y reversible del flujo aéreo documentada por espirometría. Los medicamentos que se utilizan para tratar a los pacientes asmáticos se dividen en dos grupos: de alivio y controladores. Como representantes de los fármacos de alivio, tenemos agonistas B2 de acción corta, anticolinérgicos y corticosteroides sistémicos, mientras que los controladores son principalmente agonistas B2 de acción prolongada y glucocorticoides inhalados. Consideraciones finales: El asma es una condición que afecta la vida diaria del paciente. Su fisiopatología está relacionada con la producción de citocinas inflamatorias que desencadenan un ataque de asma, las cuales se clasifican y tratan al analizar el cuadro clínico y la espirometría.

Palabras clave: Asma, Obstrucción, Exacerbación.

\section{INTRODUÇÃO}

A asma pode ser definida como uma condição causada por broncoconstrições episódicas e reversíveis, secundária a inflamação da via aérea, que pode ocorrer por vários fatores, como infecções, alérgenos ambientais e irritantes. É uma condição imunomediada e multifatorial que se apresenta com uma clínica bastante variada e complexa (PATEL SJ e TEACH SJ, 2019).

A asma é uma das afecções respiratórias mais frequentes do mundo, responsável por estar presente em 8,2\% da população dos Estados Unidos da América (EUA), tendo 1 criança acometida a cada 12 nascidas. No Brasil, de acordo com a Sociedade Brasileira de Pneumologia e Tisiologia (SBPT), a asma está presente em torno de 20 milhões de brasileiros, é responsável por aproximadamente 350.000 internações anualmente e é a terceira causa de hospitalização no Sistema Único de Saúde (SUS). Felizmente, devido a um maior entendimento dessa condição respiratória, o número de internações por asma no Brasil caiu $49 \%$ na última década (BOULET LP, et al., 2019).

Sua apresentação clínica é restrita, porém bastante complexa, e é caracterizada primariamente pela presença de dispneias episódicas e sibilância, que são geralmente relatadas pelos pacientes como falta de ar e chiado no peito (pilares clínicos da asma). Além disso, podemos ter outros sinais clínicos relacionados a limitação crônica da via aérea como a presença de baqueteamento digital em adultos, e diminuição da velocidade de crescimento em crianças (PATEL SJ e TEACH SJ, 2019)

A fisiopatologia da asma é bastante rica e bem delimitada na literatura médica. É uma condição imunomediada de amplo componente genético que tem como principal protagonista as reações de hipersensibilidade que envolvem a Imunoglobulina $E(\lg E)$, a qual desencadeará uma cascata de reações 
inflamatórias que levam a degranulação de mastócitos e, consequentemente, a uma resposta patológica exacerbada que afetará diretamente o fluxo aéreo de forma de reversível (PELAIA C, et al., 2020)

O diagnóstico da asma é clínico, comumente feito a partir dos 2 anos de idade; seu tratamento é abundantemente protocolado em guidelines, cujo mais conhecido é a Global Initiative for Asthma 2021 (GINA). Ela descreve de forma objetiva e clara a sua abordagem, classificação, bem como a intervenção a ser realizada de acordo com a classificação risco do paciente e sua etiologia. Como principal componente diagnóstico desta condição, temos a história clínica e a espirometria, que são um divisor de águas para efetivar o diagnóstico e excluir outras causas confundidoras de dispneias crônicas, enquanto temos os broncodilatadores como principais fármacos para controle de sintomas. (BOULET LP, et al., 2019)

Esse trabalho visou angariar as mais recentes e atualizadas bibliografias que giram em torno da abordagem geral da asma, englobando sua fisiopatologia, manifestações clínicas, fatores de risco, métodos diagnósticos, bem como sua intervenção terapêutica. Com isso, ele objetivou elucidar, bem como facilitar a compreensão das informações que tangem à asma.

\section{REVISÃO BIBLIOGRÁFICA}

Para fins didáticos, esta revisão optou pela divisão propedêutica da asma em quatro planos: fisiopatologia, destacando as principais moléculas e vias inflamatórias associadas à asma, o quadro clínico da doença em questão, seu diagnóstico e classificação pelos critérios mais atualizados, bem como seu manejo terapêutico farmacológico e não farmacológico, incluindo as condutas de resgate.

\section{Fisiopatologia}

A asma é uma doença imunomediada, inflamatória e altamente complexa, caracterizada por uma obstrução reversível e intermitente da via aérea baixa devido a contração do músculo liso bronquial em resposta a um gatilho ambiental ou a uma infecção de via aérea superior. As principais teorias fisiopatológicas da asma respaldam a inflamação mediada por citocinas (atopia) como principal componente do processo de doença (LAMBRECHT BN, et al., 2019).

A maioria dos pacientes asmáticos são atópicos e apresentam um padrão alérgico de inflamação das vias aéreas. A inflamação alérgica é comandada pelos linfócitos T auxiliares CD4 + (Th2), os quais secretam interleucina (IL)- 4, IL-5 e IL-13, e são algumas vezes referidos como asma alérgica, enquanto os pacientes que não apresentam este tipo de inflamação são classificados como asma não alérgica (LOUREIRO CC, et al., 2018).

Essas interleucinas, uma vez liberadas, se ligam aos seus receptores, liberando histamina, prostaglandinas e leucotrienos que, por sua vez, irão estimular a produção de lgE, mastócitos, basófilos e eosinófilos. Essa resposta inflamatória de forma crônica irá causar uma lesão irreversível com estreitamento da via aérea e acometimento do sistema mucociliar. Terapias recentes interrompem a via inflamatória inibindo a ação de mediadores específicos (IgE e IL-5) (BARNES PJ, 2017).

Esse recrutamento de células inflamatórias para a via aérea é justificado por um componente fisiopatológico presente na maioria dos pacientes asmáticos atópicos, que é a hiper-responsividade brônquica (HRB), caracterizada por uma tendência do músculo liso bronquial de contrair em resposta a um alérgeno inalado, o qual irá causar um estreitamento agudo e transitório do brônquio, reversível por meio de broncodilatadores até o momento que a lesão se torna intensa e há remodelamento do tecido acometido (LAMBRECHT BN, et al., 2019).

Assim, exposição a alérgenos e infecções virais da via aérea superior agem em sinergia ativando essa HRB e contribuindo pro desenvolvimento da asma. Crianças com asma ou em risco para asma, sensibilizados e expostos a alérgenos perenes (poeira, mofo, camundongo, rato ou barata) frequentemente apresentam inflamação da via aérea quando infectado pelo rinovírus. $O$ rinovírus do subtipo $C$, especificamente, em estudos recentes tem sido colocado em relação íntima com o desenvolvimento e exacerbações de asma (PATEL SJ e TEACH SJ, 2019). 
Em um exame histopatológico da mucosa brônquica, mesmo em indivíduos de asma leve e início recente, encontra-se alterações características do processo inflamatório recorrente do tecido, como infiltração da mucosa e submucosa por células inflamatórias (especialmente eosinófilos e linfócitos $T$ ), descamação irregular do epitélio, espessamento da musculatura lisa da via aérea, fibrose sub epitelial, hiperplasia de glândulas mucosas e de células caliciformes (SCHOETTLER N e STREK ME, 2019).

\section{Quadro clínico e fatores de risco}

O quadro clínico da asma é bastante claro e objetivo, seus pilares são sabidamente compostos por três sintomas: dispneia, sibilância e aperto no peito. A forma como esses sintomas se relacionam com os sinais vitais do paciente variam conforme a gravidade da asma (leve, moderado e grave), e estão intimamente ligados com os gatilhos da asma (ABUL MH e PHIPATANAKUL W, 2018).

O paciente portador de asma leve, geralmente se apresenta com dispneia restrita a atividade física, taquipneia leve, estado mental na maior parte das vezes normal, saturação maior que $95 \%$ e presença de sibilos expiratórios moderados. Já o paciente asmático moderado, comumente apresenta-se com dispneia ao falar, ortopneia, taquipneia e taquicardia moderada, pode usar musculatura acessória, sibilos difusos, estado mental agitado e saturação 91\% - 95\% (PIKE KC, et al., 2017).

O paciente grave geralmente se apresenta com dispneia em repouso, incapacidade de deitar, frequência respiratória maior que $30 \mathrm{ipm}$, usa musculatura acessória, presença de sibilos difusos tanto expiratórios como inspiratórios, frequência cardíaca maior que $120 \mathrm{bpm}$, estado mental agitado e saturação menor que 91\%. São crises que na maioria das vezes levam o paciente à emergência e há necessidade de internação para controle (PIKE KC, et al., 2017).

No que tange aos fatores de risco são vários os que estão relacionados com a patogênese da asma, e a análise desses fatores, de modo retrospectivo, permite identificá-los e estabelecer seu papel no processo de doença. Um dos principais é a Atopia, a qual está presente em cerca de $80 \%-90 \%$ dos asmáticos e possui o ácaro como principal agente responsável pela HRB (CASTILLO JR, et al., 2017).

A prematuridade também se configura como um fator de risco para asma, sendo bem prevalente nesse grupo, apesar de não se perceber nenhuma diferença de intensidade na sensibilidade atópica entre prematuros e a termo. O sexo masculino é outro fator de risco, visto que é mais prevalente em meninos por diferenças existentes entre tamanho de vias aéreas e tônus muscular (JONES TL, et al., 2018).

Outros fatores como exposição precoce à fumaça do tabaco (mães que fumaram na gestação possuem uma tendência a ter recém-nascidos, baixo peso e, consequentemente, com trato respiratório também comprometido), infecções de via aérea superior (entre elas as mais frequentes são as por vírus sincicial respiratório, adenovírus e por influenzae) e aleitamento materno não exclusivo nos primeiros 6 meses de vida também estão diretamente relacionados (FLEMING L, 2018).

\section{Diagnóstico e classificação}

O diagnóstico da asma é clínico e é feito geralmente depois dos 2 anos de idade, visto que antes dessa faixa etária utiliza-se o termo "lactente sibilante" para descrever os pacientes com o quadro clínico compatível. Em associação com a história clínica, temos a espirometria como principal exame para estratificar e classificar o paciente asmático de acordo com seu Volume Expiratório Forçado (VEF1). Feito a classificação da intensidade asmática, realiza-se o plano de tratamento direcionado e optimizado para aquele nível de gravidade (JONES TL, et al., 2018).

O passo inicial para confirmar o diagnóstico de asma consiste em uma anamnese detalhada (história pessoal e/ou familiar de asma ou atopia) incluindo triagem de sintomas respiratórios como tosse, sibilância, dispneia e aperto no peito bem como seus gatilhos. O histórico familiar é imprescindível visto que a atopia é a principal forma de asma e está presente em $80 \%$ dos pacientes. Após realização da história clínica detalhada, é fundamental um exame físico completo e direcionado para a queixa, o qual inclui obter uma espirometria pré e pós-resposta de broncodilatador (SCHOETTLER N e STREK ME, 2019). 
A asma dita leve é aquela em que os pacientes apresentam sintomas (dispneia, sibilância, aperto no peito e tosse) no máximo duas vezes por semana ou apenas durante exercícios, sendo crises de curta duração (inferior a um dia no mês) que são efetivamente resolvidas com broncodilatadores. Este nível não acomete de forma notória as atividades diárias, sem perda de mais do que um dia de trabalho/escola, sem necessidade de ir ao pronto socorro em crises e são facilmente controladas com broncodilatadores. Os valores de VEF1 antes do uso dos broncodilatadores normalmente são superiores a 90\% do Melhor Valor Pessoal (MVP) (ABUL MH e PHIPATANAKUL W, 2018).

A asma moderada é aquela na qual o paciente apresenta sintomas com frequência maior que 2 vezes por semana. A duração das crises é superior a um dia ao mês, não necessitam de corticoides sistêmicos para resolução, presença de sintomas durante a noite ocasionando o interrompimento do sono mais que duas vezes ao mês, porém não mais que duas vezes por semana. Tentativas frustradas de atividade física são bem relatadas, bem como faltas ao trabalho/escola. Esses pacientes fazem uso de broncodilatadores para alívio sintomático mais que duas vezes por semana, porém menos que duas vezes ao dia. O VEF1 geralmente está entre $50 \%-80 \%$ do MVP ou abaixo do limite inferior previsto, mas na faixa de normalidade após o uso dos broncodilatadores (WU TD, et al., 2019).

A asma considerada grave caracteriza-se pela presença de sinais e sintomas contínuos, que apresentam risco de vida/necessidade de internação durante as crises, as quais pedem por cursos recorrentes de corticosteroides sistêmicos. Os sintomas noturnos da crise, aqui, interrompem o sono mais de duas vezes por semana e prejudicam intensamente a vida diária do paciente, sendo responsável por faltas frequentes ao trabalho/escola. O uso de broncodilatadores acontece mais de duas vezes ao dia para resolução sintomática, bem como é frequente o uso de corticoide oral. O valor do VEF1 está comumente abaixo de $50 \%$ do MVP ou abaixo do limite inferior do previsto após o uso do broncodilatador (ABUL MH e PHIPATANAKUL W, 2018).

\section{Tratamento}

No que tange a abordagem terapêutica da asma, os objetivos das intervenções envolvem prevenir exacerbações, garantir a prática de exercícios físicos, erradicar possíveis efeitos colaterais dos medicamentos utilizados e, principalmente, prevenir a lesão crônica da via aérea que causaria uma possível obstrução irreversível, fenômeno intimamente relacionado com a mortalidade por insuficiência respiratória (PIKE KC, et al., 2017).

O passo inicial na abordagem interventiva do asmático é explicar de forma simples e objetiva para o responsável ou para o próprio paciente o que é e como a asma vai estar presente na vida dele. É fundamental o entendimento de que a crise asmática atua por meio de gatilhos (triggers), que podem ser dos mais variados possíveis, como alérgenos inaláveis (ácaros domésticos: $D$. pteronyssinus, $D$. farinae e a Blomia tropicalis; fungos; alérgenos eliminados de animais: gato, cão e pássaros e de baratas), Infecções de Via Aérea Superior (IVAS), mudanças abruptas do clima, exposição à fumaça de tabaco/outros irritantes, bem como estresse. Sabendo isso, quanto mais cedo os deflagradores forem identificados e evitados, melhor o prognóstico do paciente (ALWARITH J, et al., 2020).

No que diz respeito ao tratamento farmacológico da asma, é fundamental saber que a via inalatória é a mais escolhida para a administração de fármacos visto que permite a ação direta no tecido acometido. Os medicamentos usados no tratamento do paciente asmático são divididos em dois grupos: os de alívio e os controladores. Como representantes dos fármacos de alívio temos os 32 agonistas de curta duração, brometo de ipratrópio, teofilina e derivados, e corticosteroides sistêmicos, enquanto que os controladores são, principalmente, os $B 2$ agonistas de longa duração e os glicocorticoides inalatórios, e são usados de forma longitudinal e prolongada para controle da inflamação (LOUREIRO CC, et al., 2018).

Em se tratando da abordagem da crise asmática, os medicamentos B2 agonistas de curta duração são primeira linha na intervenção de alívio da agudização, bem como na prevenção da asma deflagrada por atividade física. Atuam por meio do relaxamento do músculo liso dos brônquios, protegendo as vias aéreas contra constrição, otimizando o transporte mucociliar, bem como reduzindo o extravasamento de fluido pela microcirculação. No que tange aos anticolinérgicos, temos o brometo de ipratrópio como protagonista no 
tratamento asmático, o qual será associado aos B2 de curta duração para otimizar o alívio durante crise de asma. O corticoide sistêmico é mais utilizado em pacientes que chegam na emergência com indícios de insuficiência respiratória e rebaixamento sensório (GOSENS R e GROSS N, 2018).

No que tange ao controle a longo prazo da asma, as duas principais classes de medicamentos usados são os B2 agonistas de longa duração e os glicocorticosteróides inalatórios. Tais classes são essenciais para uso longitudinal, sendo responsáveis pela redução dos sintomas diários de asma, bem como da frequência da asma noturna, melhora da função pulmonar, controle da inflamação, diminuição das flutuações de VEF1, entre outros benefícios (JONES TL, et al., 2018).

Finalmente, é imprescindível que aconteça a remoção de animais (gato, cão e outros mascotes) e dos reservatórios de seus alérgenos, do interior dos domicílios, caso sejam pacientes sensíveis a eles. No que tange ao controle de alérgenos advindos da barata, medidas como fechar bem os compartimentos de comida, manter lixeiras e ralos tampados, bem como uso de inseticidas são bastante úteis para erradicar esses alérgenos (WU TD, et al., 2019).

\section{CONSIDERAÇÕES FINAIS}

A asma é uma condição extremamente prevalente no Brasil e no mundo, responsável por um alto número de internações no SUS. É definida por broncoconstrições episódicas e reversíveis, secundária a inflamação da via aérea superior, as quais causam dispneia e sibilância crônica que repercutem diretamente na vida diária do paciente. O diagnóstico é feito primariamente através do quadro clínico e do exame de espirometria, o qual será usado para efetuar a classificação do paciente, assim como definir as linhas de tratamento. A intervenção, no geral, é feita com glicocorticóides inalatórios e broncodilatadores em alguns casos específicos. Nesse contexto, este artigo buscou efetuar um estudo abrangente sobre a asma com o objetivo de contribuir com a literatura médica.

\section{REFERÊNCIAS}

1. PATEL SJ, TEACH SJ. Asthma. Pediatrics in Review, 2019; 40(2): 549-567.

2. BOULET LP, et al. The Global Initiative for Asthma (GINA): 25 years later. Eur Respir J, 2019; 1-20.

3. PELAIA C, et al. Molecular Targets for Biological Therapies of Severe Asthma. Frontiers in Immunology, 2020; 11(1): 1-11.

4. LAMBRECHT BN, et al. The Cytokines of Asthma. Immunity Review, 2019; 50(1): 975-991.

5. LOUREIRO CC, et al. Omalizumab for Severe Asthma: Beyond Allergic Asthma. BioMed Research International, 2018; 1-11.

6. BARNES PJ. Cellular and molecular mechanisms of asthma and COPD. Clinical Science, 2017; 131(1): $1541-1558$.

7. SCHOETTLER N, STREK ME. Recent Advances in Severe Asthma: from Phenotypes to Personalized Medicine. Chest, 2019; 1-31.

8. ABUL MH, PHIPATANAKUL W. Severe asthma in children: Evaluation and management. Allergology International, 2018; 1-8.

9. PIKE KC, et al. Managing problematic severe asthma: beyond the guidelines. Arch Dis Child, 2017; 1-7

10. CASTILLO JR, et al. Asthma Exacerbations: Pathogenesis, Prevention, and Treatment. J Allergy Clin Immunol Pract, 2017; 5(4): 918-927.

11. JONES TL, et al. Diagnosis and treatment of severe asthma: a phenotype-based approach. Clinical Medicine, 2018; 18(2): 36-40.

12. FLEMING L. Asthma exacerbation prediction: recent insights. Curr Opin Allergy Clin Immunol, 2018; $18: 1-7$.

13. WU TD, et al. Asthma in the Primary Care Setting. Med Clin N Am, 2019; 103(1): 435-452.

14. ALWARITH J, et al. The role of nutrition in asthma prevention and treatment. Nutrition Reviews, 2020; 1-11.

15. GOSENS R, GROSS N. The mode of action of anticholinergics in asthma. Eur Respir J, 2018; 1-32. 